\title{
THE INFLUENCE OF THE COVID-19 PANDEMIC ON ALLOCATION OF ASSETS IN INVESTORS PORTFOLIO
}

\author{
Maroš Bobulský ${ }^{1}$, Mária Bohdalová ${ }^{2}$
}

\begin{abstract}
Investing during a pandemic is very challenging. Even in these difficult times, the investor must appropriately allocate assets into his portfolio. In this article, we discuss investing in the stock market. We are interested in creating portfolios of shares that consist of financial assets. The individual methods we use are designed to provide an allocation of funds in between individual shares. In the modern portfolio theory, the Markowitz model (Markowitz, 1952) is being used to solve these problems. The paper's main goal is to propose an efficient, robust approach to solve the Markowitz optimization problem adjusted for periods of a global decline in financial markets. In our research, we focus on robust optimization. Instead of precisely given input parameters, we propose a set of parameters from which we always select the worst possible parameter (so-called worst-case optimization). The robustness of optimization is achieved using so-called filter matrices. These matrices are used to modify historical data directly during optimization. The proposed model modifies the data by using different lengths of historical returns. Our proposed model is then compared with the original Markowitz non-robust model. We compare these two models using the properties of the second derivative of the optimization problem. Our results are visualized for different levels of investor's risk aversion. We present our methods on historical price data of five randomly selected companies traded on the US market. By comparing the proposed robust approach with the non-robust one, we show that different lengths of historical returns capture volatility changes earlier. The investor can thus reduce his risk aversion and increase his expected returns.
\end{abstract}

JEL Classification Numbers: C61, G11, G10, C65, DOI: https://doi.org/10.12955/peb.v2.248

Keywords: Markowitz model, Robust optimization, Data filtering, Covid-19 crisis, Risk aversion

\section{Introduction}

The main idea of portfolio management is based on the investor who has chosen a set of financial assets or stock exchange shares from which he wants to build his portfolio. The distribution of his money among the shares is crucial for the level of his returns. How to select assets into a portfolio is described, for example, in Lan et al. (2012).

In this article, we assume that the investor has already chosen the assets, and we will solve only the allocation problem. First, the investor wants to invest the most in those shares that he considers the most profitable. On the other hand, sufficient diversification reduces the risk of sudden unexpected losses. The problem of optimal portfolio allocation is finding a compromise between increasing expected returns and reducing risk in the form of portfolio volatility. The basic optimization model often used in modern portfolio theory is known as the Markowitz Mean-Variance model (Markowitz, 1952). The formulation of this model is given in the section Theoretical background of this paper.

Our research is based on the optimal allocating of financial resources into assets from the stock market. We mainly focus on robust optimization problems that expect some uncertainty in input parameters. The main idea of robust optimization is to make input parameters more uncertain. It is done by creating an uncertainty set of parameters instead of using one specific parameter. One of the first mentions of robust optimization with uncertainty in parameters can be found in Pishvaee et al. (2011) or Mirzapour Al-ehashem et al. (2011). They dealt with optimization within the theory of supply chain management. If we talk about portfolio optimization, we can mention Tütüncü and Koenig (2004) or Boyd and Kim (2007). They used continuous sets of uncertainty for their robust calculations. The idea is that it's not easy to estimate input parameters (for example, expected returns) from historical data of asset prices. If the investor is not sure about his estimate, he can use more parameters at the same time.

This paper aims to develop a new approach for robust optimization and examine the differences in portfolio optimization models after the application of robust variations. We are looking for the optimal portfolio allocation. By optimal portfolio, we mean a portfolio that promises the highest possible expected returns with the lowest possible risk of loss. Our inputs are parameters of expected returns and correlation between assets. Input parameters are being estimated from historical data of assets prices. The optimal behavior of an effective portfolio can be described by the so-called Hamilton-JacobiBellman stochastic differential equation (i.e., HJB equation). Kilianová and Ševčovič (2013) showed

\footnotetext{
${ }^{1}$ Comenius University in Bratislava, Faculty of Management, Department of Quantitative Methods, Bratislava, Slovakia, maros.bobulsky@fm.uniba.sk

${ }^{2}$ Comenius University in Bratislava, Faculty of Management, Department of Quantitative Methods, Bratislava, Slovakia,maria.bohdalova@fm.uniba.sk
} 
how the HJB problem could be transformed into a computationally much simpler quasi-linear equation using the Riccati transformation. The secondary task that arises in the calculations is very similar in its structure to Markowitz's Mean-Variance model. Kilianová and Trnovská (2014) paid closer attention to this task. They proposed a robust variation of the deterministic approach. The vector of expected returns and the covariance matrix were gradually selected from different types of uncertainty sets. After applying robust optimization, they monitored the impact of the given approach on the portfolio distribution in comparison with the deterministic approach. We followed up on this work in Bobulský and Bohdalová (2019). We simulated the real-time behavior of the portfolio based on so-called filter matrices. Robust and non-robust models were compared via the Sharpe ratio. In this article, we want to show that the new proposed approach can be very useful in crisis time. In a time when the stock market decreases in its prices.

At the beginning of the Covid-19 pandemic, between February and March 2020, we could observe that almost all assets that we have used in this article started to fall in their prices. Selling the entire portfolio and buying new assets can be time and money-consuming. As a result of this problem, we started to think about the idea of how to adjust our optimization models so they can capture higher volatility in prices earlier.

In terms of the robust approach with the filter matrices, we tried to adjust our model for earlier capture of volatility in prices. Our approach is based on the principle of collecting data for various lengths of history. In the first section, Theoretical background, we discuss the formulation of robust and non-robust Markowitz-based models. These two models will be compared. Formulation of our models is based on the HJB equation and follows the article Ševčovič, Kilianová (2013). In subsection Model specification, we explain a robust approach based on so-called filter matrices. In the section Data and methodology, we provide information on the share price data we work with. We also explain the methods of numerical calculations and describe the software used to solve optimization problems. Finally, we compare proposed models in the sections Results and Discussion. We do this using the second derivative of the optimization problem, taking into account the investor's risk aversion.

\section{Theoretical background}

We start by briefly describing the basics of portfolio optimization that we use. At first, the investor chooses assets that he wants to invest in. Secondly, he has to choose how to allocate his resources to them. Allocation makes a significant difference in his final returns. Most of his money should be invested in these assets that promise the highest expected returns. On the other hand, he shouldn't forget about reducing risk by sufficient diversification.

\section{Markowitz model and robust optimization}

Some kind of equilibrium between maximum returns and minimum risk can be calculated by the Markowitz model (Markowitz, 1952):

$$
\begin{gathered}
\max _{\boldsymbol{\theta} \in \mathbb{R}^{N}} \boldsymbol{\mu}^{\boldsymbol{T}} \boldsymbol{\theta} \\
\boldsymbol{\theta}^{\boldsymbol{T}} \boldsymbol{\Sigma} \boldsymbol{\theta} \leq \sigma^{2}, \quad \sum_{i=1}^{N} \theta^{i}=1, \quad \boldsymbol{\theta} \geq 0,
\end{gathered}
$$

where vector $\boldsymbol{\theta} \in R^{N}$ is the output variable. It is the vector of allocation that we are looking for. We denote by $\sigma^{2}$ a priori chosen parameter for maximal portfolio volatility. Greek letters $\boldsymbol{\mu}$ and $\boldsymbol{\Sigma}$ refer to the vector of expected returns and covariance matrix, respectively. These are our input parameters that need to be estimated from historical data.

This optimization problem maximizes the expected return with respect to the condition that the portfolio's volatility can't exceed some a priori chosen level. We assume that the investor wants to invest all of his money, and therefore, the sum of elements of the $\boldsymbol{\theta}$ vector is equal to one. Only buying shares is allowed (all elements of $\boldsymbol{\theta}$ should be greater than zero or equal to zero). Short transactions are not allowed.

The problem defined by (1)-(2) is a convex optimization problem. Some methods, how to solve this problem can be found, e.g., in Boyd, Vandenberghe (2004). 
We start with the estimation of the input parameters $\boldsymbol{\mu}$ and $\boldsymbol{\Sigma}$. The transformation to a robust optimization is based on the fact that these parameters are not easy to estimate. For instance, an average of historical returns can be used to estimate the vector of expected returns. However, the problem arises when we choose the right length of historical data from the past. If we use history that is too long, the average can be calculated from values that no longer correspond to the economic conditions on the market. If we use too short history of data, the resulting average is not reliable. Robust optimization is based on the fact that if we cannot determine the parameters accurately, we create a set of several estimates, from which the model selects the worst possible one. We call this set the set of uncertainty.

Formula (3) shows a possible robust modification of optimization problem (1):

$$
\max _{\boldsymbol{\theta} \in \mathbb{R}^{N}} \min _{\mu \in \mathrm{M}} \boldsymbol{\mu}^{T} \boldsymbol{\theta}
$$

In this case, we don't have an exactly calculated vector of expected returns $\boldsymbol{\mu}$, but we have some set of parameters M instead. Robust optimization means that at every point of maximization of expected return, we take the worst possible parameter value from the set of parameters $M$. This method is also called worst-case optimization. Several possible ways to produce sets of uncertainty can be found in Kilianová, Trnovská (2014).

This paper considers uncertainty in the covariance matrix of asset returns, not in expected returns. The covariance matrix of returns refers to the correlation and volatility of assets.

\section{Model Specification}

Kilianová and Ševčovič (2013) considered the problem of portfolio optimization by solving the Hamilton-Jacobi-Bellman (HJB) equation. The HJB is a stochastic differential equation that describes the evolution of a portfolio in time. In their article, they dealt with the solution of this equation. The HJB equation is difficult to solve. In their paper, they proposed its transformation to a simpler quasilinear equation, and they used Riccati transformation. The resulting solution eventually contains a secondary partial problem that is remarkably similar to Markowitz's mean-variance model. We can write this problem as:

$$
\alpha(\varphi)=\min _{\theta \in \Delta^{n}}\left(-\boldsymbol{\mu}^{\boldsymbol{T}} \boldsymbol{\theta}+\frac{\varphi}{2} \boldsymbol{\theta}^{\boldsymbol{T}} \boldsymbol{\Sigma} \boldsymbol{\theta}\right)
$$

Where $n$ is the number of assets and $\Delta^{n} \in R^{n}$ is compact convex set defined as:

$$
\Delta^{n}=\left\{\boldsymbol{\theta} \in \mathbb{R}^{n} \mid \theta^{i} \geq 0, \sum_{i=1}^{n} \theta^{i}=1\right\},
$$

where $\varphi$ is coefficient of investor's risk aversion and $\alpha(\varphi)$ is a utility function.

Our research model is based on the formula (4). Note that our model solves a very similar problem as the Markowitz model. We minimize portfolio volatility and reverse return at the same time. In addition, this formulation allows us to use a new parameter $\varphi$, which represents the investor's risk aversion. The greater this aversion, the more attention is paid to the member with volatility during optimization. Volatility can be understood as a measure of risk.

$$
\varphi(x)=1-\frac{\boldsymbol{U}^{\prime \prime}(x)}{\boldsymbol{U}^{\prime}(x)}
$$

Where, $\boldsymbol{U}$ is the investor's utility function, and the letter $x$ refers to the logarithmic volume of his money. Robust form of the formula (4) can be written as:

$$
\alpha(\varphi)=\min _{\theta \in \Delta^{n}}\left(-\boldsymbol{\mu}^{\boldsymbol{T}} \boldsymbol{\theta}+\frac{\varphi}{2} \max _{\mathrm{A} \in \mathbb{P}} \boldsymbol{\theta}^{\boldsymbol{T}} \boldsymbol{\Sigma}^{\boldsymbol{A}} \boldsymbol{\theta}\right)
$$

Where, $\mathbb{P}$ is the uncertainty set of modifications of covariance matrix $\boldsymbol{\Sigma}$.

Bobulský (2018) showed that optimization formula (7) can be rewritten as:

$$
\min _{\theta \in \Delta^{n}}\left\{-\boldsymbol{\mu}^{\boldsymbol{T}} \boldsymbol{\theta}+\max _{\mathbf{A} \in \mathbb{P}} \frac{\varphi}{2} \boldsymbol{\theta}^{\boldsymbol{T}} \mathbf{X}^{\mathbf{T}} \mathbf{A}^{\mathbf{T}} \mathbf{Q} \mathbf{A X} \boldsymbol{\theta}\right\}
$$


Where, $\mathbf{X}$ is a matrix that contains historical returns. Every column represents one company or share. Matrix $\mathbf{A}$ is the so-called filter matrix that was introduced in Bobulský (2018). Filter matrices are matrices of zeroes and ones. Every row consists of only one value 1, while other elements of the row are equal to zero. The number of columns is greater than the number of rows. It means the length of the new filtered data set $\mathbf{Y}=\mathbf{A X}$ is shortened. The matrix $\mathbf{Q}$ is defined as:

$$
\mathbf{Q}=\frac{1}{m} \mathbb{I}-\frac{1}{m^{2}} \mathbf{1 1}^{T}
$$

Where $\mathbb{I}$ is the identity matrix, $\mathbf{1}$ is a vector with all elements equal to one, and $m$ denotes the number of rows of the filter matrix $\mathbf{A}$.

We get different covariance matrices $\boldsymbol{\Sigma}$ for different types of filtrations (different matrices $\mathbf{A}$ ). The set of matrices $\mathbf{A}$ creates an uncertainty set.

The last necessary step, before using our model, is the appropriate choice of matrices A. One possible option is, for example, skipping some days. One matrix $\mathbf{A}$ would be designed to take returns from even days, the other matrix $\mathbf{A}$ would take returns from odd days. The filtering, selected in this way, would be sufficient to create uncertainty in the input parameter $\boldsymbol{\Sigma}$. Our goal is to design filter matrices to create uncertainty. We assume that the proposed model responds to market volatility changes better and prevents unexpected losses better than the original Markowitz model.

Now we decided to design the data filtering by the way when the elements of the matrix $\mathbf{A}$ take historical returns from intervals of different lengths into the past. We present filtering that calculates volatility from the last two to four days as an example (we present one covariance matrix $\boldsymbol{\Sigma}$ for each given data length). In this way, we get three filter matrices:

$$
\begin{aligned}
\mathbf{A}_{1} & =\left[\begin{array}{lllllllll}
0 & 0 & 0 & 0 & 0 & 0 & 0 & 1 & 0 \\
0 & 0 & 0 & 0 & 0 & 0 & 0 & 0 & 1
\end{array}\right] \\
\mathbf{A}_{2} & =\left[\begin{array}{lllllllll}
0 & 0 & 0 & 0 & 0 & 0 & 1 & 0 & 0 \\
0 & 0 & 0 & 0 & 0 & 0 & 0 & 1 & 0 \\
0 & 0 & 0 & 0 & 0 & 0 & 0 & 0 & 1
\end{array}\right] \\
\mathbf{A}_{3} & =\left[\begin{array}{lllllllll}
0 & 0 & 0 & 0 & 0 & 1 & 0 & 0 & 0 \\
0 & 0 & 0 & 0 & 0 & 0 & 1 & 0 & 0 \\
0 & 0 & 0 & 0 & 0 & 0 & 0 & 1 & 0 \\
0 & 0 & 0 & 0 & 0 & 0 & 0 & 0 & 1
\end{array}\right]
\end{aligned}
$$

The number of columns in matrices $\mathbf{A}$ is adjusted according to the length of the original data.

\section{Data and methodology}

Let our portfolio is composed of five companies whose shares are traded on the US market:

- Apple Inc.

- Microsoft Corporation

- Walmart Inc.

- AT\&T Inc.

- The Coca-Cola Company.

We used the sample of data that consists of the daily returns of individual assets. The analyzed data period is one year and starts from July 1, 2019 and ends on June 30, 2020. We downloaded the share prices from Yahoo finance. Then we calculated daily returns as the logarithm of the current price ratio to the price of the previous day.

We create the uncertainty set of matrices $\mathbf{A}$ similarly as in the previous section. We chose historical data intervals of 2, 4, and 6 weeks. Each filter matrix refers to one interval length. Our set of uncertainty is not continuous, it is discrete. For a discrete type of uncertainty, our task can be rewritten in the following form:

$$
\begin{gathered}
\min _{\theta \in \Delta^{n}}\left\{-\boldsymbol{\mu}^{\boldsymbol{T}} \boldsymbol{\theta}+\frac{\varphi}{2} \delta\right\} \\
-\delta+\boldsymbol{\theta}^{\boldsymbol{T}} \mathbf{X}^{\mathbf{T}} \mathbf{A}^{\mathbf{T}} \mathbf{Q} \mathbf{A} \mathbf{X} \boldsymbol{\theta} \leq 0, \quad \forall \boldsymbol{A} \in \mathbb{P}
\end{gathered}
$$


Problem (10)-(11) can be solved numerically. For calculations, we use CVX software, which is being used to solve convex optimization problems. CVX is a free extension of Matlab.

We solve the problem in a robust and non-robust way. We then compare the results. Robust optimization uses selected filter matrices A. The non-robust model (original Markowitz) is obtained by omitting matrices $\mathbf{A}$ (i.e., $\mathbb{P}=\mathbb{I}$ ).

\section{Results and Discussion}

As we have already mentioned, our approach is based on collecting data from time periods with different lengths of the past. We assume that long history data are good for estimating correlations more precisely. On the other hand, short history data are better for earlier capture of higher volatility. Our robust model is a combination of both approaches.

Figure 1 shows the composition of the portfolio according to the risk aversion $\varphi$. The results presented in Figure 1 were computed on daily returns from January 15, 2020 to February 28, 2020 (at volatile times at the beginning of the Covid-19 crisis). Each color refers to the share of one company, and in total, all shares must add up to number one. We can see that the larger the risk aversion is, the more companies are included in the portfolio. We can see in Figure 1 that the robust model applies higher diversification sooner. We can see it in the left shift of dashed lines.

We use the second derivative of the optimization problem (7) in the variable of risk aversion $\varphi$ to compare robust and original non-robust models. It can be shown (Kilianová, Ševčovič, 2013) that for optimization problem (7) the first derivative in variable $\varphi$ is:

$$
\alpha^{\prime}(\varphi)=\frac{1}{2} \widehat{\boldsymbol{\theta}}^{\boldsymbol{T}} \boldsymbol{\Sigma} \widehat{\boldsymbol{\theta}}
$$

The second derivative $\alpha^{\prime \prime}(\varphi)$ can be easily calculated numerically.

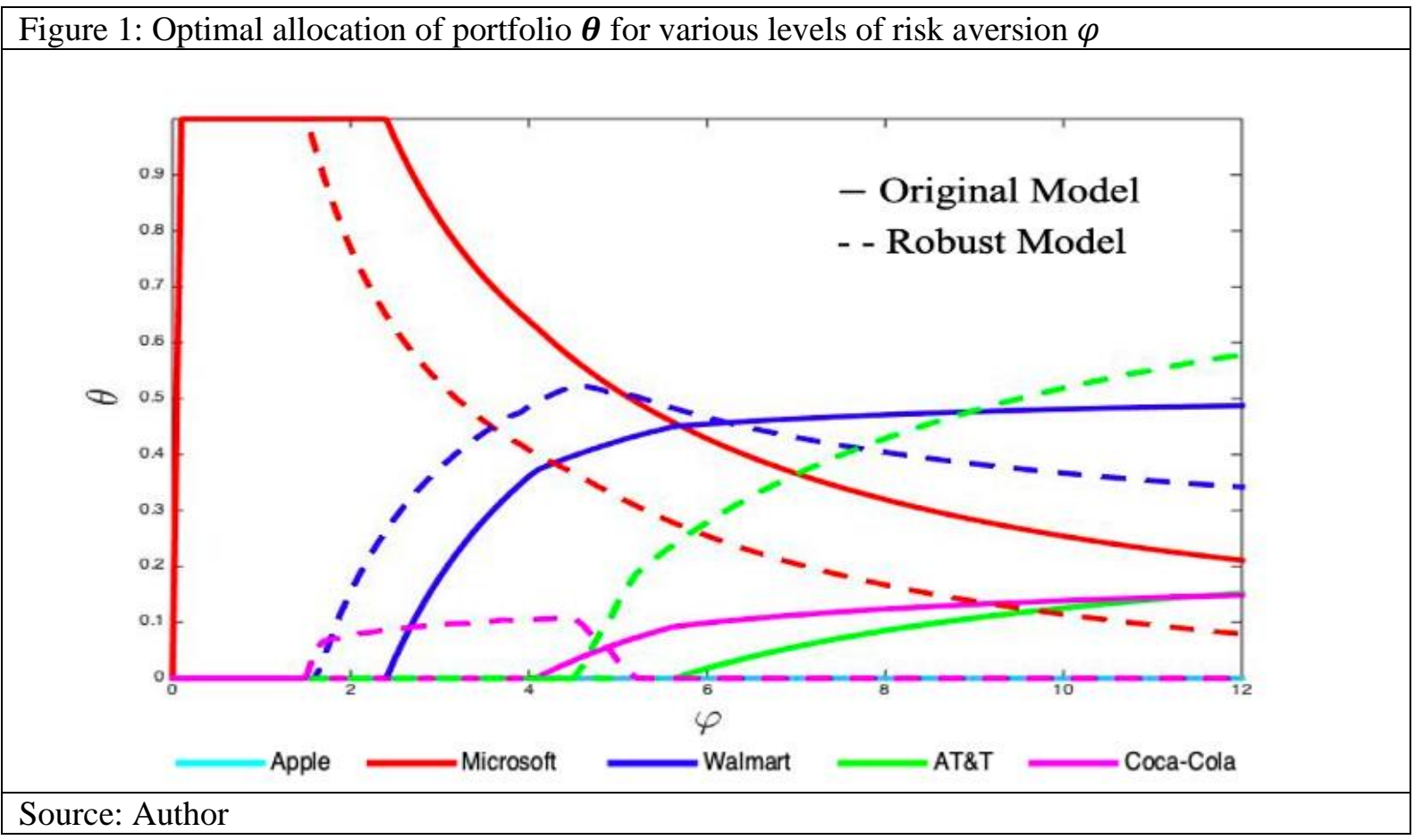

The second derivative in risk aversion is related to portfolio distribution, and points of discontinuity refer to changes in portfolio composition. We compare plots of these derivatives for different models to observe the differences in portfolio diversification. We want to show that our robust optimization approach increases diversification in portfolio automatically during times of global market downturn, but, on the other hand, behaves similarly to the original non-robust approach during times of low volatility (e.g., at times of steady increase in asset prices). It means the investor can manually decrease his risk aversion $\varphi$ since he knows that robust optimization will adjust the risk aversion of the model to his wished level of $\varphi$ during a crisis. 
Figure 2: The second derivative $\alpha^{\prime \prime}(\varphi)$. Analyzed data period: January 15, 2020 to February 28, 2020, after start of the Covid-19 crisis

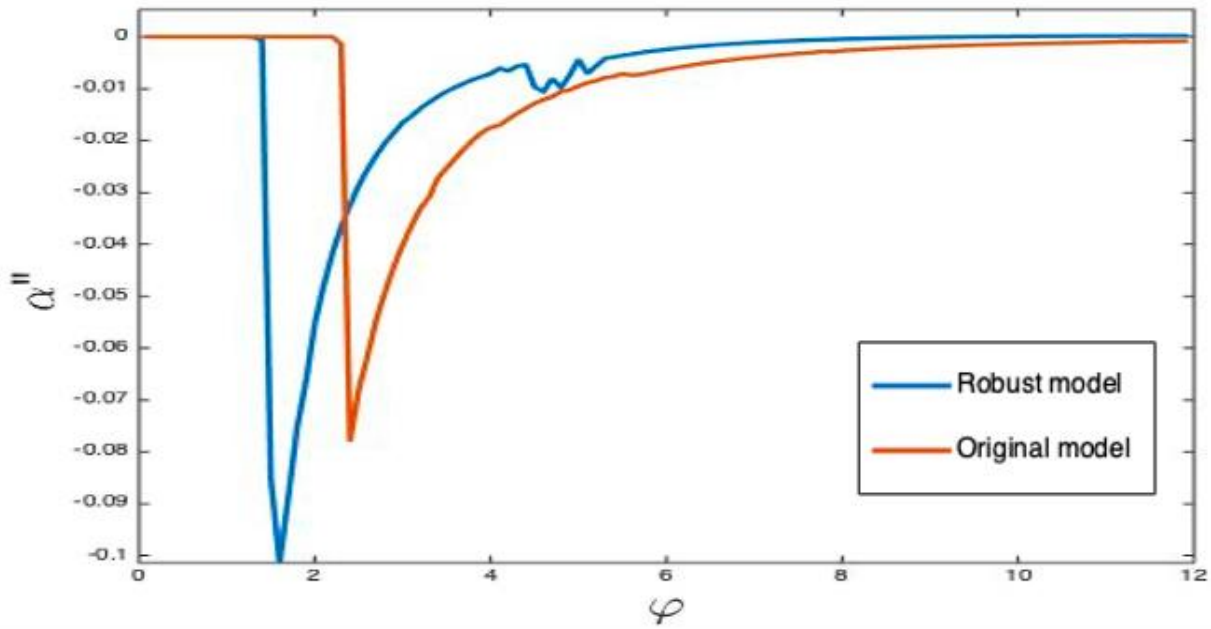

Source: Author

Figure 2 shows plots of the second derivatives $\alpha^{\prime \prime}(\varphi)$ for robust (blue) and original non-robust (orange) models computed on daily returns from January 15, 2020 to February 28, 2020 (volatile times at the beginning of the Covid-19 crisis). Points of discontinuity refer to changes in portfolio composition in Figure 1. The blue line represents the left shift of points of discontinuity for the robust approach.

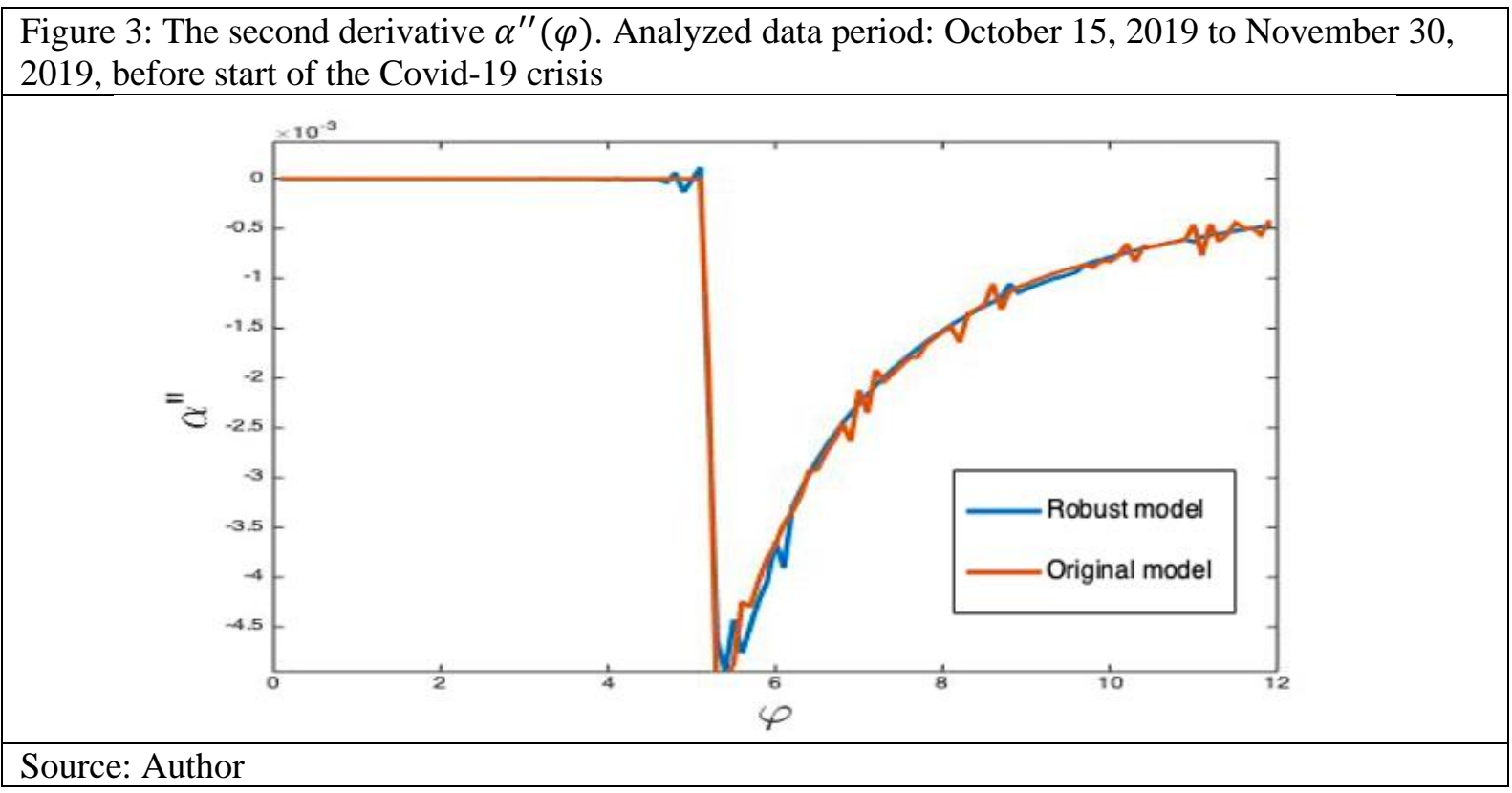

We can compare Figure 2 and Figure 3, only time periods were changed. Figure 2 shows our results for unstable times influenced by the beginning of the Covid-19 crisis. Figure 3 shows our results over a stable period with low volatility. Figure 3 shows plots of the second derivatives $\alpha^{\prime \prime}(\varphi)$ for robust (blue) and original (orange) models computed on daily returns from October 15, 2019, to November 30, 2019, during time with lower volatility. Points of discontinuity show similar behavior in portfolio composition for our two models.

\section{Conclusion}

According to the results shown in Figure 2 and Figure 3, we propose investing strategy that allows investors to manually decrease risk aversion so that during the Covid-19 crisis, the two graphs overlap and the two models behave similarly. This modification has a positive impact on expected returns during the times before the start of the Covid-19 crisis. 
It can be seen from Figure 2 that a suitable shift for this particular case would be to reduce the risk aversion of the robust model by one unit. Graphs of the second derivatives after adjustment are shown in Figure 4.

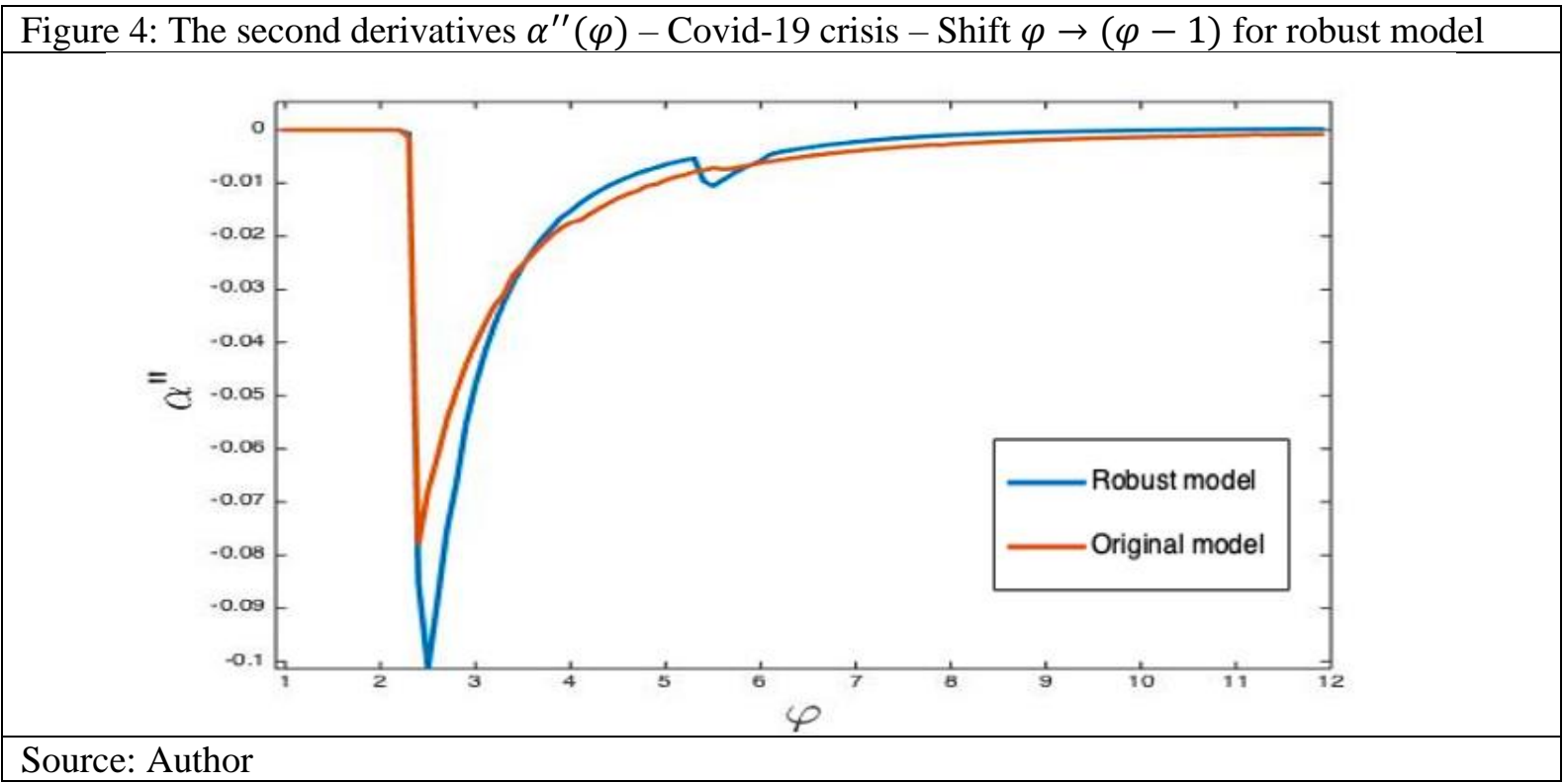

Figure 4 shows plots of the second derivative for robust model (blue) with shifted $\varphi \rightarrow(\varphi-1)$ and original model (orange) computed on daily returns from January 15, 2020 to February 28, 2020.

Points of discontinuity refer to similar behavior of portfolios for various levels of $\varphi$. Table 1 shows expected returns calculated for two reference periods (before the start of the Covid-19 crisis (Figure 1) and after the start of the Covid-19 crisis (Figure 2)).

\begin{tabular}{|c|c|c|c|}
\hline & Expected returns (p.a.) & Before crisis & Covid-19 crisis \\
\hline & Original model $(\varphi=6)$ & $5.67 \%$ & $-2.17 \%$ \\
\hline & $\begin{array}{l}\begin{array}{l}\text { Robust model with } \varphi \text { shifted } \\
(\varphi=5)\end{array} \\
\end{array}$ & $5.85 \%$ & $-2.22 \%$ \\
\hline
\end{tabular}

As we can see, the robust model offers higher expected returns in times without any crisis. It is caused by lower risk aversion that leads to lower diversification. The investor can take this risk because, as we can see in the second column, the robust model will adjust automatically. The losses (negative expected returns) will be similar to the original non-robust model during the crisis.

This article shows that the robust model uses a higher degree of diversification in times of high volatility. On the other hand, before the crisis, we were able to show that a robust and non-robust approach gave similar results. If the investor is satisfied with having his returns similar to other investors during a crisis, it allows him to use less diversification in times without a crisis. In our example, we reduced $\varphi$ by one. In practice, it is possible to calculate the value of the shift by simulating the beginning of the crisis and then estimating the required shift. Based on these simulations, it is possible to propose an accurate exact procedure for estimating risk aversion shift in the future.

\section{Acknowledgment}

This work was supported by the Faculty of Management, Comenius University in Bratislava, Slovakia.

\section{References}

Bobulský, M. (2018). Properties of the value function of a parametric quadratic programming problem. Comenius University in Bratislava, Master thesis, pp. 1-55.

Bobulský, M., Bohdalová, M. (2019). Robust Optimization Methods in Modern Portfolio Theory. European Financial Systems 2019: Proceedings of the 16th International Conference, Brno, Masaryk University, pp. 49-56.

Boyd, S., Kim, S.J. (2007, November). Robust Efficient Frontier Analysis with a Separable Uncertainty Model

Boyd, S., Vandenberghe, L. (2004). Convex Optimization, Cambridge University Press, New York 
Kilianová, S., Ševčovič, D. (2013). A Transformation Method for Solving the Hamilton-Jacobi-Bellman Equation for a Constrained Dynamic Stochastic Optimal Allocation Problem. The ANZIAM Journal, 55(1), pp.14-38. doi:10.1017/S144618111300031X.

Kilianová, S., Trnovská, M. (2014). Robust portfolio optimization via solution to the Hamilton-Jacobi-Bellman equation. International Journal of Computer Mathematics, vol. 93(5), pp. 725-734, https://doi.org/10.1080/00207160.2013.871542.

Lan, W., Wang, H., Tsai, Ch. L. (2012). A Bayesian Information Criterion for Portfolio Selection. Computational Statistics \& Data Analysis, vol. 56(1), pp. 88-99, https://doi.org/10.1016/j.csda.2011.06.012.

Markowitz, H. (1952). Portfolio Selection. The Journal of Finance, 7, pp. 77-91.

Mirzapour Al-e-hashem, S.M.J., Malekly, H., Aryanezhad, M.B. (2011). A multiobjective robust optimization model for multi-product multi-site aggregate production planning in a supply chain under uncertainty, Int. J. Prod. Econ. 134, $28-42$. Pishvaee, M.S., Rabbani, M., Torabi, S.A. (2011). A robust optimization approach to closed-loop supply chain network design under uncertainty, Appl. Math. Model. 35, pp. 637-649.

Tütüncü, R., Koenig, M. (2004). Robust asset allocation. Ann. Oper. Res. 132, pp. 157-187.

http://blogs/psychologytoday.com/blog/digital-children 